\title{
Revisión de las tablas de composición de alimentos usadas para estimar la ingesta de nutrientes en Ecuador
}

\author{
Revision of food composition \\ tables used to estimate \\ nutrient intake in Ecuador
}

\begin{abstract}
Tables and food composition databases (FCT/FCDB) are tools used for dietetic evaluation. Objective: to identify different FCT used as an instrument to estimate the nutrient intake in Ecuador. The collection of data was made through key informants -self completed questionnaires-, supplemented with institutional web research. An analysis of content was performed to the identified FCT/FCDB by general and methodological variables. Referenced tables were: Ecuadorian-1965 (70\%), INCAP (60\%), México (60\%). 7 FCT and 3 FCDB were reported, 8 from Latin America and 2 from Spain. One table was constructed by direct method (Ecuadorian-table), 7 by indirect, and 1 had no information. 6 FCT/FCDB defined the nutrients, 9 expressed values per (100g/ edible portion). In order to asses a proper ingest in Ecuador, it is necessary to have an updated ecuadorian FCT, Ecuadorian-1965 shows few analyzed foods and, not updated analytic techniques. The authors recommend the usage of FCT-INCAP, due to its food list reflects similarities with ecuadorian foods. In addition, INCAP disposes of a FCDB in which ecuadorian recipes can be included. Key words: ecuadorian foods; food composition tables; food composition databases; nutrients intake; food consumption; systematic errors.
\end{abstract}

\section{INTRODUCCIÓN}

Las Tablas de Composición de Alimentos (TCA) son herramientas para la valoración dietética y del estado nutricional de individuos y grupos poblacionales. Se definen como una recopilación de datos de la composición química de los principales alimentos disponibles y representativos en un determinado ámbito geográfico (1). Son importantes en la ciencia de la nutrición y la salud porque a partir de ellas, se obtiene el contenido energético y las cantidades de macro y micronutrientes presentes en los alimentos. En el ámbito de la salud pública permiten determinar las ingestas recomendadas por edad y país; así como establecer relaciones causales entre nutrientes y estado de salud y con marcadores bioquímicos.
Rocío Ortiz-Moncada $(1,2)$

Karina Rocha G. (1)

Gloria Domenech A. (1)

(1) Escuela de Nutrición y Dietética, Facultad de la Salud, Universidad Técnica de Manabí, Ecuador (2) Grupo de Investigación de Alimentación y Nutrición. Facultad Ciencias de la de la Salud, Universidad de Alicante, Ecuador

Dirigir la correspondencia a: Profesora

Rocío Ortiz Moncada Departamento Enfermería Com. Med. Preventiva y Salud Pública e Historia de la Ciencia. Edificio de Ciencias Sociales Universidad de Alicante.

Campus de Sant Vicent del Raspeig. Ap.99. E-03080 Alacant Telf: +34965903400 Ext. 2632 Fax: +34965906439 e-mail: rocio.ortiz@ua.es

Este trabajo fue recibido el 21 de Noviembre de 2015 y aceptado para ser publicado el 15 de Abril de 2016.

En la nutrición clínica son importantes en los tratamientos y manejo en dietoterapia y soporte nutricional (2).

Pese a la utilidad de las TCA en la valoración de la ingesta de nutrientes, la literatura científica reporta fuentes de error por factores de variabilidad en la elaboración de las mismas. En esta variabilidad se encuentra la complejidad en los factores intrínsecos del alimento relacionados con la variabilidad genética ya sea animal o vegetal; el grado de maduración; los contenidos y tipo de nutrientes del suelo; el grado de fertilización; las condiciones climáticas; la tecnología industrial y doméstica y los factores de tipo anatómico o morfológico, entre otros (3). Todos éstos ocasionarán variabilidad en los componentes específicos o nutrientes del alimento a analizar $(4,5)$. 
Además de los errores mencionados, existen otras fuentes de error derivadas de aspectos metodológicos en la elaboración de las propias TCA. Entre ellos se relacionan, los factores analíticos, los factores de estandarización, problemas en la codificación de los alimentos, errores en la terminología para expresar algunos nutrientes y alimentos y, la diversidad de las fuentes de información entre otros (3). Los relacionados con los factores analíticos son importantes a tener en cuenta, ya que dependiendo del tipo de prueba analítica utilizada, se determina un nutriente específico u otros, que no es el esperado. Por ejemplo, en la determinación de proteínas totales, utilizando la determinación de nitrógeno total, ésta incluye tanto sustancias proteicas como nitrogenadas no proteicas, lo que conduce a sobrevalorar el contenido total de proteínas. Utilizar el método Soxhlet frente a métodos de hidrólisis previa, conduce a una infravaloración en el contenido de grasa (4).

La literatura señala que para evitar los errores metodológicos citados, las TCA deberían de contener en el contenido de la tabla la descripción metodológica relacionada con la identificación del alimento, el registro de recogida, descripción detallada del alimento recogido y la manipulación en el laboratorio (6).

Por lo tanto, uno de los primeros pasos para realizar un estudio epidemiológico nutricional y valorar la ingesta de nutrientes, consiste en seleccionar una TCA que minimice los sesgos y errores que limitan la validez externa e interna de los estudios. Su información sería considerada esencial al elegir una TCA para evaluar ingesta de nutrientes de población en riesgo nutricional de determinados nutrientes y para mejorar la asociación entre el indicador de la dieta y otros indicadores.

En la actualidad se desconoce cuáles son las TCA que se utilizan en Ecuador en los estudios epidemiológicos y sus limitaciones metodológicas. Este artículo tuvo como objetivo identificar las diferentes tablas de composición de alimentos que se emplean como instrumento para estimar la ingesta de nutrientes en población ecuatoriana.

\section{MATERIAL Y MÉTODOS}

Estudio de revisión documental de las tablas de composición de alimentos empleadas en el Ecuador. Con ello se pretendió examinar el grado, el alcance y la naturaleza de las TCA utilizadas en los diferentes campos académicos y de investigación. La población de estudio fue constituida por TCA indicadas como las que se utilizaban en estudios poblacionales de ingesta de nutrientes en el Ecuador. Para identificarlas se partió de fuentes secundarias -base de datos de instituciones ecuatorianas-y de información obtenida de fuentes primarias -entrevistas a informadores clave (IC).

En el proceso de selección de los organismos públicos participantes como fuente de datos secundaria, se utilizó como criterio de inclusión que la misión institucional estuviera estrechamente relacionada con la alimentación. De esta manera, se incluyeron el Ministerio de Salud Pública del Ecuador (MSP) y el Ministerio de Inclusión Económica y Social (MIES) $(7,8)$. Sin embargo, aquellas instituciones que su misión está relacionada con la salud, pero no así con programas de nutrición, por ejemplo, la Agencia Regional de Control Sanitario (ARCSA) y el Instituto Ecuatoriano de Normalización (INEN), fueron excluidas del estudio $(9,10)$. Se identificaron TCA a través de la red de bibliotecas virtuales de la Universidad Técnica de Manabí (11) utilizando el buscador Academic Search y la base de datos EBSCO Information Service. Se realizó la búsqueda a través de los sitios web de las instituciones gubernamentales que cumplieron los criterios de inclusión, utilizando como palabra clave "tabla de composición de alimentos" y otras palabras clave asociadas a TCA en español e inglés ( tabla 1, parte B). En los sitios web de los Ministerios de Salud Pública y de Inclusión Económica y Social, se buscaron los programas relacionados con TCA y alimentación $(12,13)$.

Referente a las fuentes de información primaria, los informadores clave (IC) fueron seleccionados de universidades públicas y privadas que impartían la Carrera de Nutrición en sus diferentes denominaciones en el Ecuador $(n=12)$; para evitar conflicto de intereses se excluyó la Universidad Técnica de Manabí. Los nombres y afiliaciones de los IC se obtuvieron de la web oficial de la Secretaría Nacional de Educación Superior Ciencia y Tecnología (SENESCYT) (14). Las universidades incluidas en el estudio fueron: Pontificia Universidad Católica del Ecuador, Universidad Internacional del Ecuador, Universidad San Francisco de Quito, Universidad Católica de Santiago de Guayaquil, Escuela Superior Politécnica del Litoral, Universidad de Guayaquil, Universidad de Especialidades Espíritu Santo, Escuela Superior Politécnica del Chimborazo, Universidad de Cuenca, Universidad Técnica del Norte, Universidad Estatal de Milagro, Universidad Técnica de Babahoyo. También se incluyeron las instituciones gubernamentales (Ministerios de Salud y de Inclusión Económica y Social).

Para recoger la información de las instituciones universitarias, se enviaron cartas de invitación vía correo electrónico y en algunos casos, se complementó con entrevista directa durante el mes de Agosto del 2014 por dos investigadoras (RO, JR). En estas cartas, se especificó el objetivo de la investigación y la confidencialidad en los datos. A estas cartas se anexó un cuestionario que contenía la siguiente pregunta semiestructurada: En los estudios epidemiológicos sobre consumo de alimentos que usted consulta durante sus actividades de docencia, prácticas, investigación e intervención entre otros, ¿Qué tablas de composición de alimentos utiliza? La pregunta con 5 opciones de respuesta fueron seleccionadas por el equipo investigador considerando las tablas indicadas como las más usadas. Los IC debían de seleccionar con una X la TCA empleada. Las opciones de respuesta fueron las siguientes: 1. Tabla de composición de alimentos ecuatorianos (1965); 2. Tabla de composición de alimentos de LATINFOODS (FAO América latina); 3. Tabla de Composición de Alimentos de Centroamérica/INCAP 4. Tabla de Composición de Alimentos de México; 5. Otras tablas. Además, debían de indicar la forma de presentación de las mismas: 1. Impresa, 2. Sistematizada (programa informático), 3. Internet. También se solicitó como requisito, indicar la referencia completa del libro, cuestionarios o links utilizados. Algunas universidades respondieron más de un cuestionario con más de un informador clave. En estos casos, se unificaron las opciones que reportaban la misma repuesta en esa institución.

Para lograr identificar las TCA de fuentes secundarias y primarias que posteriormente se van a recuperar, se hace necesario definir las características de una TCA.

Según la literatura, una tabla de composición de alimentos y una base de datos de composición de alimentos (BDCA) tienen el mismo contenido -datos sobre composición química de alimentos- pero, distinto formato. Las tablas tiene un formato impreso, mientras que las bases de datos de nutrientes tiene un formato electrónico. Las tablas son libros y por tanto, la modificación de las mismas se realiza tras la recopilación de nuevos datos. Las BDCA son, por el contrario, mucho más flexibles. Se pueden modificar de inmediato y añadirles datos analíticos de alimentos que acaban de ser analizados, recalculados o corregidos con un nuevo factor de conversión. Estos 
cambios, en el caso de las TCA, tienen que esperar hasta una nueva edición (4).

Con las TCA y BDCA se cuantificaron en tipo y en número las tablas de composición y bases de datos de alimentos usadas en el país. A partir de la identificación de las TCA y BDCA incluidas, se recuperaron los documentos en texto o programas informáticos utilizando la plataforma de Google Académico y finalmente con informadores clave. Se elaboró un protocolo ad hoc de recogida de información, el cual contenía las siguientes variables categorizadas en dos grupos, generales y metodológicas, según reporta la literatura científica $(1,3,15,16)$ :

Grupo de variables generales: Nombre de la TCA; Procedencia (país de publicación); Datos de publicación (1. Año de publicación, 2. Número de edición, 3. Editorial); Tipo de publicación (se refiere a la institución que elaboró la TCA: 1. Pública, 2. Privada, 3. Mixta).

\section{Grupo de variables metodológicas}

- Método de construcción de la TCA, hace referencia a la descripción de los métodos utilizados para la construcción de la TCA: 1. Directo (análisis bromatológico directo para la determinación de nutrientes), 2 . Indirecto (recopilación de datos existentes -publicaciones científicas, empresas alimentarias, laboratorios de control de alimentos y de TCA publicadas-), 3. Combinado (fusión de métodos directos e indirectos) (15, 4). NA (no aplica porque no se dispone de información).

- Tipo de muestreo: (probabilístico y no probabilístico (17), se aplica solo a TCA que hayan utilizado método directo, que requiere plan de muestreo representativo de alimentos consumidos en una zona geográfica $(15,18)$ ): 1. Muestreo aleatorio simple; 2. Muestreo aleatorio-sistemático; 3. Muestreo aleatorio-estratificado; 4. Muestreo por conglomerados; 5. Muestreo no-probabilístico; 6. NA (no aplica).

- Lugar de provisión de alimentos: (lugar de donde provienen los alimentos de la muestra de los métodos directo o combinado): 1. Nivel nacional; 2. Nivel regional; 3. Nivel provincial; 4. Nivel cantonal; 5. NA (no aplica).

- Contenido de la TCA: 1. Valores de composición (cuerpo central, formado por las tablas en sí que contiene: descripción del alimento, de los nutrientes y valores de concentración entre otros); 2. Información auxiliar necesaria para facilitar y usar de forma correcta las TCA, contienen: Texto preliminar (nutriente incluidos y forma de expresión, forma de describir los alimentos, estructura de grupos y subgrupos de alimentos y recomendaciones de uso correcto TCA); idealmente una TCA debería de contener tanto los valores de composición como información auxiliar (15). Por ello, la escala de la variable se determinó de la siguiente manera:

1. Valores de composición + información auxiliar; 2. Valores de composición; 3. Información auxiliar.

- Definición de nutrientes: se refiere a la definición adecuada de los nutrientes teniendo en cuenta sus características físico-químicas o fisiológicas para evitar fuentes de error (15). Escala de la variable: 1. Si define; 2. No define.

- Descripción del alimento: la descripción adecuada del alimento es importante debido a la gran variedad y géneros existentes, a las diferentes preparaciones culinarias y procesos industriales, a la adición o sustracción de algún ingrediente o nutriente entre otros (15). Escala de la variable: 1. Si describe; 2. No describe.

- Estructura de las TCA: grupos y subgrupos de alimentos, útil para facilitar la búsqueda y clasificación de los alimentos (1). Escala de la variable: 1. Grupos + Subgrupos + Alimentos; 2. Grupos + Alimentos; 3. Alimentos.
- Porción comestible: valores de expresión del contenido de nutriente por porción comestible o medida casera común (3). Escala de la variable: 1. Valores expresados por $100 \mathrm{~g}$. de porción comestible; 2. Valores expresados por $100 \mathrm{~g}$ de porción comestible + ración media habitual; 3. Valores expresados para ración media habitual; 4. NA (no especifica).

Se realizó un análisis de contenido de las TCA y BDCA indicadas por los IC utilizando un cuestionario de variables generales y metodológicas de las TCA tal como indica la bibliografía $(1,3,15,16)$.

\section{RESULTADOS}

La tabla 1 muestra las tablas de composición de alimentos (TCA) que se obtuvieron a partir de las fuentes secundarias. En ella se indican las instituciones consultadas, el sitio web, criterios de búsqueda utilizados y los resultados obtenidos. La parte A se refiere específicamente a los resultados de los organismos gubernamentales, donde no se encontró ninguna referencia de TCA o BDCA. La parte B explica los resultados de búsqueda de la red de bibliotecas virtual de la UTM, donde se encontraron 96 documentos utilizando las palabras clave. Sólo 1 documento cumplió con los criterios de inclusión definido como BDCA (19).

La tabla 2 relaciona los resultados de las tablas de composición de alimentos que los IC registraron a partir de fuentes primarias. Se envió un total de 14 cuestionarios (12 a universidades y 2 a instituciones gubernamentales). Se obtuvo una tasa de respuesta de $71,4 \%$ ( $n=10$ instituciones y 14 IC). Respondieron 9 universidades (13 IC) y 1 institución gubernamental (1 IC). Los IC que respondieron referenciaron 11 TCA en diferentes formatos y 2 BDCA. El 70\% de las instituciones indico que la TCA ecuatorianos, 1965, (formato impreso) (16) es la más utilizada $(n=7)$, a ésta le siguen las de Centroamérica/INCAP (20) (formato impreso) $(n=6)$ y las TCA de México (internet) (21) $(n=6)$. La menos referenciada es la LATINFOODS FAO (América Latina) web-virtual (internet) (22). Entre las otras TCA utilizadas por los IC está la Tabla Peruana de Composición de Alimentos (internet) (23), la cual es la más usada (20\%). Las otras TCA utilizadas, sólo fueron reportadas por una universidad, correspondiente a la TCA CESNID (impresa) (1), tabla de calorías de Neil Stevens (internet) (24), TCA ecuatorianos del 2010 (formato Excel), Instituto Nacional del Niño y la Familia -INFA- (impresa), Instituto de Economía Local y Solidaria (impresa) y la de la Tesis doctoral de $M^{a}$ Gabriela Barrezueta Roldan (impresa). Otras bases de datos como BDCA referidas a la Tabla de Composición Química de Alimentos Chilenos (internet) (25) y a la TCA Colombianos (internet) (26).

De los 13 resultados obtenidos, se recuperaron 9 documentos, 3 no se lograron recuperar en formato papel o en digital y del restante (Tablas de LATINFOODS, FAO-América Latina), no se consideró porque no se obtuvo en formato especifico ya que estas tablas están incluida en la página web de la FAO (27).

Finalmente se procedió al análisis de los 9 documentos recuperados de fuentes primarias. En la tabla 3 se muestra las características generales y metodológicas de las TCA recuperadas en formato impreso, obtenidas tanto de fuentes secundarias (1 base de datos de composición de alimentos: NUTRIPLATO) (28), como primarias (7 TCA + 2 BDCA). En relación a las características generales, se destaca que 8 TCA provienen de países de Suramérica y 2 TCA de España, 7 fueron elaboradas por organismos públicos y 4 por organismos privados. 
De las variables metodológicas, se destaca que una fue elaborada por método directo, 7 por indirecto y 2 no disponen de información. La TCA ecuatorianos 1965, es la única elabo- rada con método directo, con un muestreo no probabilístico, y la mayoría de los alimentos provenían de un solo sector del país, cumplimentándose con recolecciones regionales. Las

\section{TABLA 1}

Tablas de composición de alimentos obtenidas de fuentes secundarias. Ecuador 2014.

A: Instituciones gubernamentales Institución

Ministerio de Salud Pública

\section{Sitio web \\ www.msp. gob.ec} Fecha de acceso 29/08/2014

www.mies. gob.ec Fecha de acceso29/08/2014

inisteriode Inclusión Económica Social

B: Universidad Técnica de Manabí Institución

Bibliotecas virtuales

\section{Sitio web}

www.utm. edu.ec http://www. utm.edu.ec/bibliotecavirtual.asp EBSCO InformationService

Fecha de acceso29/08/2014
Criterio de búsqueda

Palabras clave: Tablas composición de alimentos (TCA)

1)Programas yservicios

2)Programa alimentarionutricional integrat PANI

3)Programa de provisiónde alimentos

Criterio de búsqueda Palabras clave: 1) Tablas de composición de alimentos ecuatorianos 2) Tablas de alimentos ecuatorianos

3)Alimentosecuatorianos

4) Ecuadorianfoodcompositiontables

5) Ecuadorianfoodtables

\section{No hayreferencia a TCA*ecuatorianos en introducción ni en metodología http://goo.gl/RBvUug \\ Tampoco en ENSANUT-ECU** 2011-2013. http://goo.gl/LsCl5a}

No se hace referencia a TCA ${ }^{*}$, solo habla de ejes del proyecto, sin mayor detalle ni links de enlace http://goo.gl/OnQDdP

No se obtiene respuesta al enlace http://goo.gl/NVZ9hc

No se obtiene respuesta al enlace http://goo.gl/Afyo4K

Resultados 1) 50 resultados, 1 artículo cumple criterio
inclusión:

2) 66 resultados, 1 articulo cumple criterio inclusión 3) 1 resultado, 1 artículo cumple criterio

4) 96 resultados, 1 articulo cumple criterio inclusión

5) 96 resultados, 1 artículos cumple criterio inclusión

* Tabla de composición de alimentos.

** Encuesta Nacional de Salud y Nutrición.

\section{TABLA 2}

Tablas de composición de alimentos de uso común referenciadas por instituciones de educación superior y gubernamentales. Ecuador 2014.

Universidades ecuatorianas e Institución gubernamental

Pontificia Universidad Católica del Ecuador

U. Internacional del Ecuador

U. San Francisco de Quito

U. Católica de Santiago de Guayaquil

Escuela Superior Politécnica del Litoral

U. Especialidades Espíritu Santo

Escuela Superior Politécnica del Chimborazo

U. de Cuenca

U. Estatal de Milagro

Ministerio Inclusión Económica y Social

Total $(n=10)(100 \%)$

a Tablas peruanas de composición de alimentos

'Tesis doctoral de Ma. Gabriela Barrezueta Roldan

- Tabla de composición química de alimentos chilenos

'Centre d'Ensenyament Superior de NutricióiDietètica.

'Tabla de composición de alimentos colombianos

tTabla de composición de alimentos ecuatorianos, revisada en 2010

${ }^{9}$ Tabla calorías de Neil Stevens

${ }^{h}$ Instituto Nacional del Niño y la Familia

i Instituto de Economía Local y Solidaria

\begin{tabular}{|c|c|c|c|c|}
\hline \multirow{4}{*}{ Ecuatoriana, 1965} & \multicolumn{4}{|c|}{ Tablas y bases de composición de alimentos(TCA y BDCA) } \\
\hline & LATINFOODS & INCAP & México & Otras \\
\hline & & $x$ & $x$ & $\stackrel{x}{\operatorname{TPCA}^{a}}$ \\
\hline & & $x$ & & $\stackrel{x}{\text { TD MG Barrezueta }}$ \\
\hline$x$ & & $x$ & $\mathrm{x}$ & $\begin{array}{c}\mathrm{x} \\
\text { TCQACH }^{\mathrm{c}}\end{array}$ \\
\hline$x$ & & & $x$ & $\stackrel{x}{\mathrm{CESN}^{\mathrm{d}}}$ \\
\hline$x$ & $x$ & & & 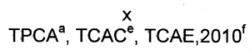 \\
\hline$x$ & & $x$ & $x$ & \\
\hline$x$ & $x$ & $x$ & $x$ & $\stackrel{x}{T^{g}}$ \\
\hline$x$ & & $x$ & $x$ & $\stackrel{x}{I^{h}}$ \\
\hline $\mathrm{x}$ & & & & $\stackrel{x}{x}$ \\
\hline $7(70 \%)$ & $3(30 \%)$ & $6(60 \%)$ & $6(60 \%)$ & \\
\hline
\end{tabular}


tablas elaboradas bajo el método indirecto recopilan la mayoría de sus datos de la National Nutrient Database for Standard Reference (USDA) (29).

En relación a la información auxiliar sobre el uso de las TCA/BDCA, así como el contenido de la tabla, 8 documentos disponen de la información y 2 (TCA ecuatorianas, 1965 y 2010) no presentan información sobre el manejo de la tabla. En relación a la definición de los nutrientes, 6 TCA/BDCA disponen de información clara y detallada, mientras que 4 no tienen información. Todas las TCA/BDCA hacen una clara diferenciación entre las diversas partes de los alimentos e indican también si es crudo o procesado y el tipo de proceso al que fue sometido. En cuanto a los grupos y subgrupos de alimentos, 9 TCA/BDCA están organizadas de esta forma $y$, refieren los valores por $100 \mathrm{~g}$ de porción comestible.

\section{DISCUSIÓN}

Los resultados del estudio revelan 13 tablas y bases de composición de alimentos disponibles para valorar la ingesta de nutrientes según referencian instituciones gubernamentales y educativas del Ecuador. La mayoría de las TCA y BDCA (80\%) consultadas provienen de países de Suramérica y las otras de España, siendo la Tabla de Composición de Alimentos Ecuatorianos del 1965 (TCAE-1965), la más referenciada, seguidas de las del INCAP y de México. Sin embargo, en el análisis de variables metodológicas de las TCA recuperadas, se destaca que sólo la TCAE-1965 utilizó un análisis bromatológico directo para determinar nutrientes. La mayoría utilizan método indirecto y otras no lo especifican, presentan heterogeneidad en la información auxiliar de referencia en la bibliografía original, técnicas analíticas empleadas, origen y número de muestras de alimentos entre otros, lo que conduce a errores sistemáticos en la estimación de nutrientes.

Un estudio realizado en Brasil (30), analizó información de fuentes y metodologías utilizadas en la determinación de contenido de vitamina A y hierro en diferentes TCA y programas de Software reportadas en literatura científica, con el fin de determinar el mejor recurso de evaluación para estudiar deficiencias nutricionales. Sus resultados reportaron un total de 10 TCA y 5 programas de Software (BDCA). Resultados similares fueron encontrados en el presente estudio, a pesar que el objetivo de revisión fue diferente, 11 TCA y 2 BDCA. Así mismo, coincide en la utilización de la TCA del INCAP como una de las más empleadas por su utilidad en la determinación en la ingesta de nutrientes.

En este estudio, los resultados muestran dificultad en el acceso público a información y recuperación de documentos de TCA en diferentes formatos en los organismos públicos gubernamentales consultados. Este hecho podría estar evidenciando limitaciones de orientación en la política para la construcción, uso y acceso a las TCA a nivel nacional. En este sentido y siendo una de las funciones de la política pública orientar en procesos metodológicas homogéneos que permitan realizar estudios de investigación e intervención que faciliten el dialogo entre los profesionales, la comunidad y el gobierno, se presenta esto como una limitación. La alta respuesta de los IC de las instituciones para reportar las TCA usadas en la valoración de la ingesta de nutrientes, muestra una participación activa y

\section{TABLA 3}

Características generales y metodológicas de las tablas de composición de alimentos identificadas por informadores clave de instituciones universitarias y gubernamentales. Ecuador 2014.

\begin{tabular}{|c|c|c|c|c|c|c|c|c|c|c|}
\hline \multicolumn{3}{|c|}{ Característicasgenerales } & \multicolumn{8}{|c|}{ Caracteristicasmetodológicas } \\
\hline $\begin{array}{l}\text { Tabla composición alimentos y base de } \\
\text { datos de alimentos (TCA y BDCA) }\end{array}$ & $\begin{array}{c}\text { País } \\
\text { procedencia }\end{array}$ & $\begin{array}{l}\text { Tipopublica } \\
\text { ción }\end{array}$ & $\begin{array}{c}\text { Método } \\
\text { construcción }\end{array}$ & $\begin{array}{c}\text { Tipo } \\
\text { muestreo }\end{array}$ & $\begin{array}{l}\text { Lugar } \\
\text { provisión } \\
\text { alimentos }\end{array}$ & $\begin{array}{l}\text { Contenido } \\
\text { TCA }\end{array}$ & $\begin{array}{l}\text { Definición } \\
\text { nutriente } \\
\text { (Si/No) }\end{array}$ & $\begin{array}{l}\text { Descripción } \\
\text { alimento } \\
\text { (Si/No) }\end{array}$ & $\begin{array}{l}\text { Estructura } \\
\text { TCA }\end{array}$ & $\begin{array}{l}\text { Valores/ } \\
\text { porción } \\
\text { comestible }\end{array}$ \\
\hline $\begin{array}{l}\text { TCA Ecuatoriano. 1965. } 1^{\text {a }} \text { Ed. } \\
\text { MSP-IN. Ecuador }\end{array}$ & Ecuador & Pública & Directo & No-Probb & Nacional & Val Com ${ }^{c}$ & No & $\mathrm{Si}$ & $G^{e}+S^{1}+A^{\theta}$ & $100 \mathrm{~g}$ \\
\hline INCAP. 2007. 2a Ed.INCAPIOPS & Guatemala & Pública & Indirecto & $\mathrm{NI}^{\mathrm{a}}$ & $\mathbf{N} l^{\mathbf{a}}$ & $\operatorname{Val} C^{c}+1 A^{d}$ & $\mathrm{Si}$ & $\mathrm{Si}$ & $G^{e}+S^{\prime}+A^{0}$ & $100 \mathrm{~g}$ \\
\hline $\begin{array}{l}\text { TCA México. 2010. } 2^{\mathrm{a}} \text { Ed. } \\
\text { Mc Graw Hill }\end{array}$ & México & Privada & Indirecto & $\mathrm{NI}^{\mathrm{a}}$ & $\mathrm{N}^{\mathbf{a}}$ & $V C^{c}+I A^{d}$ & $\mathrm{Si}$ & $\mathrm{Si}$ & $G^{0}+S^{f}+A^{9}$ & $100 \mathrm{~g}$ \\
\hline $\begin{array}{l}\text { Tablas peruanas de composición de } \\
\text { alimentos, } 2009.8^{\mathrm{a}} \text { Ed. } \\
\text { Instituto Nacional de Salud. } \\
\text { Ministerio de Salud de Perú. }\end{array}$ & Perú & Publica & Indirecto & $\mathrm{NI}^{\mathrm{a}}$ & $\mathrm{Nl}^{2}$ & $V C^{c}+I A^{d}$ & No & $\mathrm{Si}$ & $G^{e}+S^{\prime}+A^{0}$ & $100 \mathrm{~g}$ \\
\hline $\begin{array}{l}\text { TCA del CESNID (Centre } \\
\text { d'Ensenyament Superior de } \\
\text { NutricióDietètica). 2004. } 2^{\mathrm{a}} \text { Ed. Mc } \\
\text { Graw Hill }\end{array}$ & España & Privada & Indirecto & $\mathrm{NI}^{\mathrm{a}}$ & $\mathrm{Ni}^{\mathrm{a}}$ & $V C^{c}+I A^{d}$ & $\mathrm{Si}$ & $\mathrm{Si}$ & $G^{e}+S^{f}+A^{g}$ & g \\
\hline $\begin{array}{l}\text { Tabla de calorias de Neil Stevens. } \\
\text { 2011. } 2^{\mathrm{a}} \text { Ed. Sirio }\end{array}$ & Argentina & Privada & Indirecto & $\mathrm{Nl}^{\mathrm{a}}$ & $\mathrm{NI}^{\mathrm{a}}$ & $V C^{C}+I^{d}$ & No & $\mathrm{Si}$ & $G^{e}+S^{f}+A^{g}$ & $100 \mathrm{~g}$ \\
\hline $\begin{array}{l}\text { TCA ecuatorianos, revisada en } 2010 . \\
\text { No dispone información }\end{array}$ & Ecuador & Pública & $\mathrm{NI}^{\mathrm{a}}$ & $\mathrm{NI}^{\mathrm{a}}$ & $\mathrm{NI}^{\mathrm{a}}$ & $v c^{c}$ & $\mathrm{Si}$ & $\mathrm{Si}$ & $G^{e}+S^{\prime}+A^{g}$ & $\mathrm{NI}$ \\
\hline $\begin{array}{l}\text { Tabla de composición química de } \\
\text { alimentos chilenos. } 1992 . \\
\text { Universidad de Chile }\end{array}$ & Chile & Publica & Indirecto & $\mathrm{NI}^{\mathrm{a}}$ & $\mathrm{Nl}^{\mathrm{a}}$ & $V C^{c}+I A^{d}$ & No & $\mathrm{Si}$ & $G^{e}+S^{t}+A^{0}$ & $100 \mathrm{~g}$ \\
\hline $\begin{array}{l}\text { TCA colombianos, } 1978 . \\
\text { Instituto Colombiano de Bienestar } \\
\text { Familiar. } 2010\end{array}$ & Colombia & Publica & $\mathrm{NI}^{\mathrm{a}}$ & $\mathrm{Nl}^{\mathrm{a}}$ & $\mathrm{Nl}^{\mathrm{a}}$ & $V C^{c}+1 A^{d}$ & $\mathrm{Si}$ & $\mathrm{Si}$ & $A^{0}$ & $100 \mathrm{~g}$ \\
\hline $\begin{array}{l}\text { BDCA NUTRIPLATO. } 2010 . \\
\text { Universidad de Córdoba. España. }\end{array}$ & España & Publica & Indirecto & $\mathrm{NI}^{\mathrm{a}}$ & $\mathrm{NI}^{\mathrm{a}}$ & $V C^{c}+1 A^{d}$ & $\mathrm{Si}$ & $\mathrm{Si}$ & $G^{e}+S^{f}+A^{a}$ & $100 \mathrm{~g}$ \\
\hline $\begin{array}{l}\text { a No dispone de información } \\
\text { "No probabilistico(No-Prob) } \\
\text { 'Valores de composición(VC) } \\
\text { d Información auxiliar (IA) } \\
\text { e Grupo (G) } \\
\text { 'Subgrupo (S') } \\
\text { 'Alimentos (A) }\end{array}$ & & & & & & & & & & \\
\hline
\end{tabular}


compromiso por parte de estos actores para aportar resultados durante el proceso de la investigación basada en su práctica.

En el análisis de contenido sobre características generales de las TCA referenciadas por los IC, los resultados muestran un hilo conductor con lo reportado en la literatura relacionado con el uso de TCA propias del país para estimar ingesta de nutrientes. La literatura reporta que lo ideal es que cada país tenga un programa establecido para la gestión de sus propios datos de composición de alimentos y que los considere un recurso nacional público tan importante como cualquier otra colección nacional de datos (6). Es así, que en varios estudios de estimación de ingesta alrededor del mundo, se comprueba que se utilizan las TCA del propio país (31-34). En el presente estudio, a pesar de la diversidad de TCA, la mayoría proceden de Latinoamérica, lo que muestra una selección apropiada de una TCA nacional o provenientes de la región Suramericana.

La literatura científica indica escasos estudios originales realizados en Ecuador para valorar ingesta alimentaria, en los que las TCA usadas no son las de Ecuador o no se especifica la TCA que se aplica $(19,35,36)$. A modo de ejemplo, en el estudio de evaluación nutricional de la dieta ecuatoriana, los autores no utilizaron una TCA específicamente ecuatoriana, sino que diseñaron un software "Nutriplato" basado en datos de tablas del USDA y LATINFOODS, para determinar la composición nutricional de los platos (19). Es importante resaltar que LATINFOODS, no es específicamente una TCA, sino que es una compilación de datos adecuados y fiables de composición de alimentos internacionales agrupados por países. LATINFOODS constituye una red mundial para promover la participación y la cooperación internacional en la difusión de los datos (27). Por esta razón, posiblemente ninguna universidad en sus respuestas señaló una TCA de un país concreto cuando se refirió a LATINFOODS. Otro estudio acerca del conocimiento y percepción del consumo de sal y el etiquetado nutricional en la población de Ecuador, Argentina y Costa Rica, no especifica la TCA utilizada para determinar la ingesta de sodio de los alimentos frescos y procesados (35).

Los estudios epidemiológicos actuales de valoración de ingesta alimentaria, se centran principalmente en la selección de instrumentos validados de recogida de información, tales como cuestionario de frecuencia de consumo, recordatorio de 24 horas entre otros, para minimizar posibles sesgos metodológicos (31-39). Sin embargo, son pocos los estudios que se centran en la selección de las TCA, a pesar que se han descrito limitaciones y sesgos en las características de las TCA para la determinación de nutrientes $(2,5,19)$. En el presente estudio se analizaron variables metodológicas de las tablas de composición de alimentos, en lo que se evidenció que en su mayoría las TCA y BDCA, utilizan el método indirecto en su construcción. Algunos autores están de acuerdo que la elaboración de una TCA es compleja y costosa, lo cual justifica la utilización de métodos indirectos $(6,18)$. A pesar que el método indirecto es el más usado según indican los datos del presente estudio, la información metodológica de procedencia y métodos analíticos de los alimentos es inexistente $y$, en otros casos ni se especifica el tipo de método. En éste sentido, estudios publicados cuestionan tanto los sesgos, como la rigurosidad y validez científica del uso de estas TCA $(2,5,30,37)$.

Para controlar los posibles sesgos del método indirecto, las tablas deberían de indicar, los datos originales de los análisis directos de donde proviene el alimento, las técnicas empleadas de muestreo por alimento, el origen de las muestras, los cálculos y ponderaciones utilizadas en las estimaciones de las concentraciones de nutrientes, para generar datos menos subjetivos y transferibles para la comunidad científica y para la población $(2,5,6)$.

La TCA ecuatoriana del año 1965, fue la única elaborada por el método directo por el Ministerio de Previsión Social y Sanidad (extinto en la actualidad) (36). En ella se presentan los resultados obtenidos en 1136 muestras recolectadas de 568 alimentos diferentes en todo el país (16). Anterior a ésta, la tabla de alimentos data de 1952 elaborada por el Instituto Nacional de Nutrición del Ecuador (36). Una de las limitaciones de la TCA del 1965, es su antigüedad, por lo tanto se cuestionan los análisis bromatológicos ya que han cambiado desde hace 50 años, al igual que las variaciones de especies y de producción de alimentos, la composición de sus nutrientes, como lo muestran algunos estudios (40). La versión actualizada del año 2010 tanto en formato impreso y digital consiste en una tabla en Excel, que carece de información general y metodológica de la procedencia de los alimentos incluidos, lo que inhabilita su uso para estudios de dieta.

Aunque el método directo es el ideal, la TCA ecuatoriana del 1965 presenta algunas limitaciones en su proceso de construcción en las técnicas empleadas de muestreo. El tipo de muestreo no probabilístico utilizado limita la inclusión de alimentos de especies representativas de todas las regiones geográficas de Ecuador (16). Para la determinación bromatológica de los nutrientes, se tomó sólo una muestra para la mayoría de los alimentos. Algunos estudios sugieren emplear técnicas adecuadas de muestreo de mas de una muestra, ya que por tratarse de materiales biológicos, un producto alimenticio muestra variaciones en su composición y, suele presentar variabilidad estadísticamente significativa $(38,41)$.

A modo de ejemplo, hay diferencias en el contenido de macronutrientes de algunas variedades menos conocidas y variedades silvestres ampliamente consumidas de arroz que puede variar desde 5 a 13\% como lo muestra el estudio realizado por Burlingame et al (2009). Los autores concluyen relevante ésta variabilidad, ya que la ingesta de una sola variedad de alimento, en lugar de otra, puede ser la diferencia entre la deficiencia de micronutrientes y la adecuación de micronutrientes (42). Por el contrario, otro estudio muestra que al analizar determinados oligoelementos en muestras de frutas y verduras cultivadas en un país como México y compararlos con los análisis descritos en tablas de composición de alimentos europeas, no hubo diferencias consistentes entre los datos mexicanos y europeos (43).

Por estas razones expuestas, los errores sistemáticos en las estimaciones de las TCA, se podrían minimizar, utilizando una base de datos común de composición de alimentos entre países para armonizar normas que mejoran la calidad de los datos (44). Los errores sistemáticos, serian entonces consistentes entre países, por lo tanto los datos serían más comparables (5). Entre las bases de datos común se pueden citar a modo de ejemplo FAO/INFOODS (27), las de United States Department of Agriculture, Agricultural Research Service -USDA- (29) y, la del -European Food Information Resource- EuroFIR que recoge información de bases de datos europeas (45). Hoy en día, estas bases de datos han cobrado mayor relevancia, por el aumento en la ingesta de alimentos frescos modificados genéticamente, de alimentos procesados, fortificados entre otros, que son de interés particular para la valoración dietética y determinantes causales de enfermedad. La necesidad de conocer la composición de nutrientes y de otros elementos que se encuentran en estos alimentos es necesaria incluirla en estas bases de datos, ya que actualmente esta información está limitada y poco disponible (46). 
Las principales limitaciones detectadas en el presente estudio están relacionadas con la recuperación de los documentos de tablas de composición de alimentos, vinculadas con las posibilidades reales para acceder a la información en internet, lo que podría llevar a un sesgo de accesibilidad (47). Para disminuir el posible sesgo citado la búsqueda se complementó con otras fuentes de información cualitativa como informadores clave y expertos.

Finalmente y con base en lo anteriormente expuesto, las autoras consideran importante realizar algunas recomendaciones a la hora de contar con una TCA o BDCA para realizar estudios de evaluación de la ingesta de nutrientes dirigida a población adulta ecuatoriana, teniendo en cuenta las variables generales y metodológicas establecidas como guía recomendada $(1,3,15,16)$. En este sentido, la TCA ideal sería aquella elaborada en el propio país de estudio y por método directo, por lo tanto, la TCA ecuatorianos de 1965, sería la más adecuada. Sin embargo, como se ha revisado anteriormente presenta sesgos que limitarían su uso (16). Al revisar las TCA elaboradas por método indirecto está la de México y la del INCAP $(20,21)$. La TCA mexicana presenta análisis de alimentos autóctonos y faltan alimentos de consumo frecuente en el Ecuador, siendo una limitación. Por tanto, las autoras aconsejan el uso de la TCA del INCAP, dada la proximidad geográfica al Ecuador y por tanto, la lista de alimentos refleja similitud. Además, adicional a la versión TCA, el INCAP dispone de una base de datos de composición de alimentos a la cual se pueden incorporar recetas ecuatorianas y obtener los datos de composición en caso de no contar con ese alimento en la BDCA. Esta BDCA del INCAP facilita el trabajo para posterior análisis de ingesta de nutrientes a partir de los alimentos consumidos provenientes de estudios para la evaluación del estado nutricional de grupos poblacionales.

\section{RESUMEN}

Las tablas y bases de datos de composición de alimentos (TCA/BDCA) son herramientas de valoración dietética. Objetivo: identificar las diferentes TCA empleadas como instrumento para estimar ingesta de nutrientes en Ecuador. La recogida de información se realizó a través de informadores-clave, cuestionarios auto-administrados, complementadas con búsquedas webs institucionales. A las TCA/BDCA identificadas se realizó análisis de contenido sobre variables generales y metodológicas. TCA referidas: Ecuatoriana-1965 (70\%), INCAP (60\%), México (60\%). Se reportaron 7 TCA y 3 BDCA, 8 Latinoamericanas y 2 Españolas. Una TCA elaborada por método-directo (ecuatoriana), 7 por método-indirecto, 2 sin-información. 6 TCA/BDCA definieron nutrientes, 9 refirieron valores $(100 \mathrm{~g} /$ porción-comestible). Para realizar una correcta estimacion de ingesta, es necesario contar con una TCA ecuatoriana actualizada. Ecuatoriana-1965 presenta pocos alimentos analizados y, métodos analíticos no-actualizados. Las autoras aconsejan el uso de TCA-INCAP, dado que la lista de alimentos refleja similitud con alimentos ecuatorianos. Además INCAP dispone BDCA para incorporar recetas.

Palabras clave: Alimentos ecuatorianos; Tabla de composición de alimentos: bases de datos de compoción de alimentos; ingesta de nutrientes; consumo de alimentos; errores sistemáticos.

\section{BIBLIOGRAFÍA}

1. Nutrition and Dietetics Higher Education Center). Tables of food composition CESNID. $2^{\circ}$ ed. Barcelona: MCGRAWHILL, 2004.
2. San Mauro I, Hernández B. Calibration tools menu and calculation of the composition nutritional food; validity and variability. Nutr Hosp. 2014; 29:929-34.

3. Mataix Verdu J, Navarro S. Tables of food composition. Mataix Verdu J. $2^{\circ}$ ed. Nutrition and food, I. Nutrients and foods. Madrid: Ergon., 2009, p. 503-7.

4. Mañas Almendros M, Martínez de Victoria Muñoz E, Yago Torregosa MD. Food composition tables and nutritional data bases. Gil Hernández A. $2^{\circ}$ ed. Nutrition treaty, II. Composition and nutritive quality of food. Madrid: Medica Panamericana., 2010, p. 615-30.

5. Merchant AT, Dehghan M. Food composition database development for between country comparisons. Nutri J [Internet]. 2006 [Access 9 Jan. 2015]; 5:1-8. Available in: http://www.nutritionj.com/content/5/1/2

6. Greenfield $H$, Southfate DAT. Food composition data, procurement, management and use, 2nd ed. Rome. United Nations Organization for Food and Agriculture (FAO), 2006 [Access 9 Jan. 2015]. Available in: http://www.fao.org/3/ contents/8ed5bc39-b8ab-501d-9fd9-be93bc933e9e/ y4705s01.pdf.

7. Ministry of Public Health, Ecuador. [Access 5 Aug. 2014]. Available in valores/misión/visión: http://www.salud.gob. ec/el-ministerio/.

8. Ministry of Economic and Social Inclusion, Ecuador. [Access 5 Aug. 2014]. Available in misión/visión/valores: http:// www.inclusion.gob.ec/misionvision/.

9. National Agency for Regulation and Control Health Surveillance. [Access 6 Aug. 2014]. Available in valores/misión/ visión: http://www.controlsanitario. gob. ec/valores/.

10. Ecuadorian Service normalization. [Access 6 Aug. 2014]. Available in valores/misión/visión: http://www.normalizacion.gob.ec/mision-vision/.

11. Technical University of Manabi. [Access 29 Aug. 2014]. Available in red de bibliotecas virtuales: http://wWw.utm. edu.ec/bibliotecavirtual.asp.

12. Ministry of Public Health, Ecuador. [Access 29 Aug. 2014]. Available in Programas y Encuesta nacional de salud y nutrición ENSANUT: http://www.salud.gob.ec/encuestanacional-de-salud-y-nutricion-ensanut/.

13. Ministry of Economic and Social Inclusion, Ecuador. [Access 29 Aug. 2014]. Available in programas y servicios: http:// www.inclusion.gob.ec/programas-y-servicios/.

14. Ministry of Higher Education, Science, Technology and Innovation, Ecuador. [Access 4 Aug. 2014]. Available in oferta académica por nivel: http://app.senescyt.gob.ec/ BuscadorOfertaAcademicaWeb/faces/index.xhtml.

15. Farrán Codina A, Zamora R. Food composition tables: public health applications. Serra Majem LI, Aranceta Bartrina J. $2^{\circ}$ ed. Nutrition and Public Health, Methods, scientific bases and applications. Barcelona. Elsevier., 2006, p. 228-33.

16. National Institute of Nutrition. Composition table Ecuadorian food. $3^{\circ}$ ed. Quito. Ministry of Social Welfare and Health, Ecuador 1965.

17. Pfizer. CICAP Clinical research in primary care. $1^{\circ}$ ed. Madrid: Luzan 5 S.A. de Ediciones., 2002.

18. Farran Codina A. Development and implementation of an information system for the production of food composition tables. Departament de Nutrition Bromatologia., Universitat de Barcelona., Barcelona-España., 2004.

19. Sánchez S, Neira JA, Pérez F, Moreno R. Preliminary nutritional assessment of the Ecuadorian diet based on a 24- $h$ food recall survey in Ecuador. Nutr. Hosp. 2013; 28:1646-56. 
20. INCAP/OPS. Food composition table Centroamerica). $2^{\circ}$ ed. Guatemala., Institute of Nutrition of Central America and Panama (INCAP)/ Pan American Health Organization (PAHO)., 2007. 2nd ed. Guatemala.

21. Muñoz de Chávez M. Food composition (nutritional value of food increased intake. $2^{\circ}$ ed., México DF. McGrawHill., 2010.

22. International Network of Food Data Systems (INFOODS). Latin American food composition tables. United Nations Organization for Food and Agriculture (FAO), [Access 18 Dec. 2014] Available in: http://www.fao.org/infoods/ infoods/tables-and-databases/latin-america/en/.

23. National Institute of Health. Peruvian food composition tables. $8^{a}$ ed. Lime. Ministry of Health., 2009.

24. Stevens N. Calories Table. Editorial Sirio S.A. $2^{\circ}$ ed., Buenos Aires., 2012.

25. Department of Food Science and Chemical Technology. Chilean Food Chemistry Composition Table. $8^{a}$ ed. Santiago de Chile. University of Chile, 1992. [Access 18 Dec. 2014]. Available in: http://mazinger.sisib.uchile.cl/repositorio/ Ib/ciencias_quimicas_y_farmaceuticas/schmidth03/index. html.

26. Colombian Institute for Family Welfare. Colombians Food Composition Table. $4^{a}$ ed. Bogotá. Ministerio de Salud Pública, 1978. [Access 18 Dec. 2014]. Available in: http:// alimentoscolombianos.icbf.gov.co/alimentos_colombianos/ acercade.asp.

27. International Network of Food Data Systems (INFOODS). INFOODS United Nations Organization for Food and Agriculture (FAO. [Access 20 Sep. 2015]. Available in: http:// www.fao.org/infoods/infoods/es/.

28. Moreno R. Nutriplato Sotfware. University of Cordoba, Córdoba-España. [Access 8 Feb. 2015]. Available in: http:// www.nutriplato.com/

29. Nutrient Data Lab. USDA National Nutrient Database for Standard Reference. National Agricultural Library/United States Department of Agriculture. [Access 20 Sep. 2015]. Available in: http://ndb.nal.usda.gov.

30. Barbosa Chagas C, Saunders C, Ferreira Campos AB, Lima Nogueira J, da Silva CL, Dutra Alves $P$, et al. Comparative analysis of vitamin $A$ and iron content in food according to different food composition tables and nutritional evaluation software programs. Food Sci. Technol Campinas. 2013; 33: 229-32.

31. Córdoba-Caro L, Luego Pérez L, García Preciado V. Nutricional adequacy of students of compulsory secondary education in Badajoz. Nutr Hosp. 2012; 27:1065-71

32. Dermience M, Mathieu F, Barthélemy JP, Maesen $P$, Romnee JM, De Maertelaer $V$, et al. The relevance of food composition data for nutrition surveys in rural Tibet: pilot study in the context of Kashin-Beck Disease. Biotechnol Agron Soc Environ. 2013; 17:32-42.

33. Parr CL, Barikmo I, Torheim LE, Ouattara F, Kaloga A,
Oshaug A. Validation of the second version of a quantitative food-frequency questionnaire for use in Western Mali. Public Health Nutr. 2002; 5:769-81.

34. Chinnock A. Development of a food frequency questionnaire and a comparison with food records. Perspect Nutr Humana. 2011;13:57-69.

35. Sánchez $G$, Peña $L$, Varea $S$, Mogrovejo P, Goetschel $M L$, Montero-Campos MA, et al. Knowledge, perceptions, and behavior related to salt consumption, health, and nutritional labeling in Argentina, Costa Rica, and Ecuador. Rev Panam Salud Pública. 2012; 32:259-64.

36. Sánchez Llaguno S. Preliminary study on the feeding of the Ecuadorian population (Department of Food Science and Food Technology). University of Cordoba, CórdobaEspaña., 2014.

37. Aparicio Vizuete A, López Sobaler AM. Basic dietetic tools for assessment nutritional status. Nutr Hosp. 2014;30:510.

38. Lazarevic K, Stojanovic D, Bogdanovic D. Estimation of Macronutrient Content in Kindergartens Meals: Food Composition Tables or Chemical Analysis?. Iran J Pediatr. 2014; 24:643-6.

39. Chinnock A. Validation of an estimated food record. Public Health Nutr. 2006; 9:934-41.

40. Davis DR, Epp MD, Riordan HD. Changes in USDA food composition data for 43 garden crops, 1950 to 1999. J Am Coll Nutr. 2004; 23:669-82.

41. Barrado R, Mayo MT, Tesedo A, Romero $H$, de la Rosa $F$. Fat composition of several "fast food". Nutr Hosp. 2008; 23:148-58.

42. Burlingame $B$, Charrondiere $R$, Mouille B. Food composition is fundamental to the cross-cutting initiative on biodiversity for food and nutrition. J Food Compost Anal. 2009; 22:361-5.

43. Sanchez-Castillo CP, Dewey PJ, Aguirre A, Lara JJ, Vaca $R$, de la Barra PL. The mineral content of Mexican fruits and vegetables. J Food Compost Anal. 1998; 11:340-56.

44. Truswell AS, Bateson DJ, Madafiglio KC, Pennington JA, Rand WM, Klensin JC. INFOODS guidelines for describing foods: A systematic approach to describing foods to facilitate international exchange of food composition data. J Food Compost Anal. 1991; 4:18-38.

45. EuroFIR -European Food Information Resource. 2015 [Access 12 Oct. 2015]. Available in: http://www.eurofir. org/?page_id=96.

46. Sichert-Hellert W, Kersting M, Chahda $C$, Schäfer R, Kroke A. German food composition database for dietary evaluations in children and adolescents. J Food Compost Anal. 2007; 20:63-70.

47. Dwan K, Altman DG, Arnaiz JA, Bloom J, Chan AW, Cronin E. Systematic Review of the Empirical Evidence of Study Publication Bias and Outcome Reporting Bias. PLoS One. 2008; 3:1-31. 